\title{
Provitamin D Doped Silica and Polymeric Films: New Materials for UV Biosensor*
}

\author{
Tatiana Orlova ${ }^{1}$, Irina Terenetskaya ${ }^{1}$, Anna Eremenko ${ }^{2}$, Natalia Surovtseva ${ }^{2}$ \\ ${ }^{1}$ Institute of Physics, National Academy of Sciences of Ukraine, Kiev, Ukraine; ${ }^{2}$ Chuiko Institute of Surface Chemistry, National \\ Academy of Sciences of Ukraine, Kiev, Ukraine. \\ Email: teren@iop.kiev.ua
}

Received August 25 ${ }^{\text {th }}$, 2010; revised October $10^{\text {th }}$, 2010; accepted October $10^{\text {th }}, 2010$.

\begin{abstract}
The original technologies of growing silica films, impregnated with 7-dehydrocholesterol (provitamin $D_{3}$ ) on quartz substrates and free transparent films on the basis of polyvinyl alcohol and polyvinyl butyral have been developed. Provitamin D photoisomerization in the films under UVB irradiation was investigated by UV absorption spectroscopy. Remarkable changes in the absorption spectrum of 7-DHC were observed in silica and polyvinyl alcohol films as compared with ethanol solution, only in polyvinyl butyral film the spectrum was very nearly, while the spectral kinetics of 7-DHC photoisomerization in all the films was different from ethanol. We suggest that several films have potential as $U V$ dosimeters to measure accumulated 'antirachitic' UV dose in the same manner as erythemic UV dose is measured by commonly used polysulphone film.
\end{abstract}

Keywords: Provitamin D Photoisomerization, Polymer Films, Silica Porous Film, UV Dosimetry

\section{Introduction}

The isomerization reactions of optically excited molecules are of considerable current interest in photochemistry. Lately many efforts went into elaboration of new materials with desirable optical properties that are based on the inclusion of photosensitive labile molecules into various organic and inorganic media (polymer films, porous materials, etc.). These guest-host materials have found wide application. They have been widely applied to heterogeneous biocatalysis, to the development of solid state optical materials [1-3]. In particular, polymer-dye films are used as the photo-active layers in light emitting diodes (LED), lasers and photovoltaic cells. Besides, porous hybrid materials find use as highly specific chemical sensors.

To design and optimize a material whose optical behavior is based on properties induced by guest molecules, it is necessary to understand how the microenvironment affects their certain dynamic properties because such processes as molecular rotations and conformation changes determine the optical behavior of the final solidstate material [4].

At a molecular level, inclusion, dissolution or adsorption processes involve direct chemical and physical in-

*DOE/GIPP/LLNL/STCU teractions between host and guest molecules, and electronic spectroscopy of specific probe molecules offers sensitive methods of reporting the local environment of the probe molecule. As an example, protein bioencapsulation has been carried out in nanoporous silica-based materials to study the biocompatibility and biological activity of proteins $[5,6]$.

In this paper we present the results of our investigations on 7-dehydrocholesterol (7-DHC, provitamin $\mathrm{D}_{3}$ ) photoisomerization in "solid" media (polymer and porous silica films). Being extension of our previous studies on the reaction medium effect on provitamin D photochemistry [7-10], this work is also directed on elaboration of new UV sensitive materials, in particular, as UV biosensors for direct monitoring of the vitamin D synthetic capacity of solar/artificial UV radiation [11,12].

\section{Materials and Methods}

The main task was to grow the UV transparent optically homogenous thin films impregnated with 7-DHC suitable for investigation of provitamin $\mathrm{D}$ photoisomerization in these films by means of UV absorption spectroscopy. Three UV transparent media have been suggested as appropriate candidates for the thin films, namely, porous sol-gel silica, polymer polyvinyl alcohol and polymer polyvinyl butyral. 


\subsection{Sol-Gel Production of Porous Silica Films Impregnated with Provitamin D}

The challenge was in finding the preparation regime of mechanically stable UV transparent homogeneous porous silica films (glasses) with sufficient content of 7-DHC entrapped within a silica matrix. First and foremost the temperature, solution $\mathrm{pH}$, particular alkoxide precursor and solvent, and their relative concentrations, should be determined to provide the optimum ratio of porosity, thickness and UV/VIS absorbance/transparency of the film. The pore size should be optimized with regard to expected suppression of conformational mobility of 7DHC (and its photoisomers) implied by their immobilization on the $\mathrm{OH}$-groups of silica surface. Furthermore, the acidity regime of sol-gel process should be carefully balanced in view of known unfavorable acid effect on 7-DHC photochemistry [13].

Next task was to assure adequate UV optical density (no less than 0.2 ) of the silica film with entrapped molecules 7-DHC, i.e., to find optimum film thickness and adequate amount of 7-DHC inside. The point is that thick films are liable to be destroyed due to collapse of the primary particles at the stage of the film heat treatment, while in thin film the amount of immobilized 7-DHC is insufficient for accurate measurements of UV absorbance.

Several approaches have been suggested for the films preparation: 1) under neutral $\mathrm{pH}, 2$ ) with solubilization of the 7-DHC molecules within $\beta$-cyclodextrin to protect molecule against the interaction with silica $\mathrm{OH}$-groups saving the conformation mobility, and 3) to introduce the solubilized molecules within mesoporous silica films via adsorption that is favorable for homogeneous distribution of 7-DHC.

The mesoporous silica films have been prepared by the sol-gel method using cationic surfactant cetyltrimethylammonium bromide (CTAB, Aldrich) as a template agent to provide the homogeneous porosity. The precursor sol was prepared by hydrolysis of TEOS in a mixture of distilled water, ethanol (Fluka), and $1 \mathrm{M} \mathrm{HCl}$ solution. Twenty-four hours after hydrolysis, the water solution of $\mathrm{CTAB}$ was added to this solution. The total molar ratios were 1 TEOS: 0.1 CTAB: $0.02 \mathrm{HCl}: 10 \mathrm{H}_{2} \mathrm{O}: 5 \mathrm{C}_{2} \mathrm{H}_{5} \mathrm{OH}$.

The material was deposited onto clean quartz substrates by the dip-coating technique. The films were dried for 12 hours at ambient temperature, followed by heat treatment at $400^{\circ} \mathrm{C}$ with a rate of $1^{\circ} \mathrm{C} / \mathrm{min}$ and calcinated at $350^{\circ} \mathrm{C}$ for $6 \mathrm{~h}$. The parameters of low-level cell of silica film were as follows: the pores diameter $3 \mathrm{~nm}$, the walls thickness $1,7 \mathrm{~nm}$, and the surface $\mathrm{S}_{\mathrm{BET}}=550 \mathrm{~m}^{2} / \mathrm{g}$.

$\mathrm{X}$-ray diffraction (XRD) of the film demonstrated 3 signals: (2 theta): 2.7: 25: 8.6, it corresponded to the interlaminar distance $34.7 \mathrm{~A}$; after $500 \mathrm{C}$ the distance was $30.4 \mathrm{~A}$, and after $600^{\circ} \mathrm{C}$ the collapse of the porous structure occurred. Hence, the film was treated at $350^{\circ} \mathrm{C}$ during 1 hour before the adsorption of 7-DHC from hexane solution, $\mathrm{C}_{7-\mathrm{DHC}}=2 * 10^{-3} \mathrm{~mol} / \mathrm{l}$.

\subsection{Preparation of Transparent Polymer Films with Entrapped 7-Dehydrocholesterol}

Two polymers - polyvinyl alcohol (PVA) and polyvinyl butyral (PVB) - were checked for the films preparation because of their rather low absorption in the spectral range of interest (260 - $300 \mathrm{~nm}$ ).

The different solubility of PVA and 7-DHC was formidable obstacle to the development of preparation technology of UV transparent optically homogenous free (without quartz substrates) PVA film impregnated with UV sensitive biomolecules (7-DHC). Since PVA is watersoluble and 7-DHC is fat-soluble, at first PVA (MW 60000) was dissolved in water to obtain $5 \%$ water solution of polyvinyl alcohol. Further ethanol solution of 7-DHC was slowly introduced into the polymer solution, which was carefully mixed, putted on the glass substrate and slowly evaporated. (The films of larger diameter $(7.5 \mathrm{~cm})$ were prepared in the Petri's vessels). Films drying procedure was accomplished in the desiccators. The critical parameters were as follows: the concentration of the initial solution of PVA in water, the drying conditions, and the appropriate concentration of 7-DHC within PVA-based film needed for the UV spectra registration. Obtained PVA films of 40-200 $\mu \mathrm{m}$ thickness were mechanically stable, colorless, but a little scattering.

To obtain better optical homogeneity we used polyvinyl butyral which can be dissolved in ethanol and thus facilitates the film preparation.

UV irradiation of the samples was performed using luminescent lamp EL-30 (280-330 nm). Spectral irradiance of the UV lamp at the sample distance $6 \mathrm{~cm}$ was determined using calibrated spectrometer EPP2000CUV + VIS (StellarNet, Inc), and the following data were obtained: $0.25 \mathrm{~mW} / \mathrm{cm}^{2}$ for the total spectral range 270-380 $\mathrm{nm}$ of El-30 lamp and $0.11 \mathrm{~mW} / \mathrm{cm}^{2}$ in the UVB spectral range.

The photoreaction course was followed by UV absorption spectroscopy, i.e., absorption spectra of the samples were recorded by Perkin\&Elmer Lambda 25 UV/VIS spectrophotometer within $230-330 \mathrm{~nm}$ before and after fixed UV exposures. In parallel, simultaneously ethanol solution of 7-DHC was irradiated in the same conditions.

\section{Results and Discussion}

Absorption spectra of silica and polymer films impregnated with 7-DHC are shown in Figure 1 in comparison with the absorption spectrum of 7-DHC in ethanol.

As one can see in Figures 1 (c, d), inclusion of 7-DHC into silica porous film and polyvinyl alcohol film sig- 
nificantly affects its absorption spectrum (that differs from well-known stability of 7-DHC spectrum in different solvents [14]), only in polyvinyl butyral film the 7-DHC absorption spectrum more closely resembles the spectrum in ethanol. The spectra in silica porous and PVA films are red shifted $(2 \mathrm{~nm})$, and the redistribution of the vibronic bands intensity slightly observed in silica film significantly increases in PVA film indicating heavy influence by the properties of the adjacent surrounding media [15].

Transformations of initial absorption spectra of the three films upon the UV lamp irradiation are shown in Figure 2 in comparison with 7-DHC in ethanol. Surprisingly, the spectral kinetics for all the films is significantly distinct from each other and from the known kinetics of 7-DHC photoisomerization in ethanol [11].

From the above spectral transformations the absorbance at $282 \mathrm{~nm}$ dependency versus UVB dose can be derived (Figure 3) that can be used for UV dosimetry.

\section{Conclusions}

Biologically effective exposures can be determined in two ways: 1) by measuring the spectral irradiance of a UV source during the period of exposure and calculating

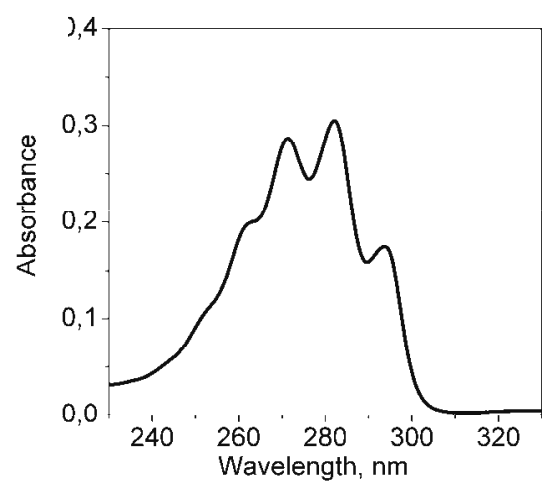

(a)

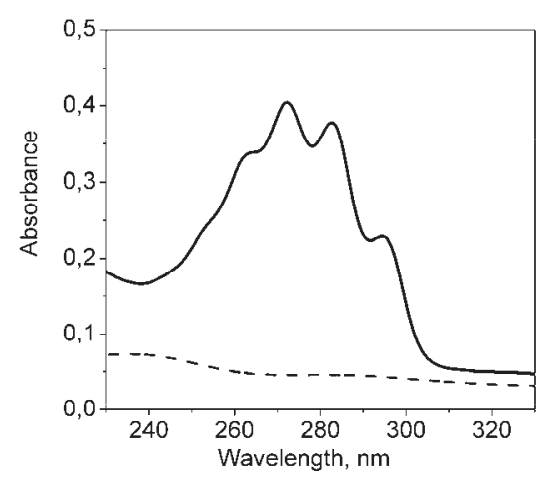

(c) the weighted integral; or 2) by using a device whose relative response to different wavelengths resembles the action spectrum for the particular photobiological effect. Hence, strict conformity between spectral sensitivity of radiation detectors and the biological action spectra that they are designed to mimic is of primary importance for adequate monitoring of a UV biologic dose. Most of broad-band UV detectors are usually designed to have a spectral sensitivity which is a close match to the CIE erythema action spectrum, e.g. polysulphone film which has a spectral response close to the erythemal response of human skin is commonly employed as film dosimeter in UV photobiology.

In view of significant difference between the CIE erythema and 'Vitamin D' action spectra [11,12], polysulphone film is incapable to adequate evaluation of the vitamin D synthetic capacity of sunlight and/or an artificial UV source. Since vitamin D synthesis is originated from the UVB photon absorption by provitamin D molecule, obviously, a UV transparent film impregnated with provitamin $\mathrm{D}$ molecules is the most appropriate material for the 'antirachitic' UV dosimetry.

This paper describes technologies of growing silicafilms, impregnated with 7-dehydrocholesterol (provita-

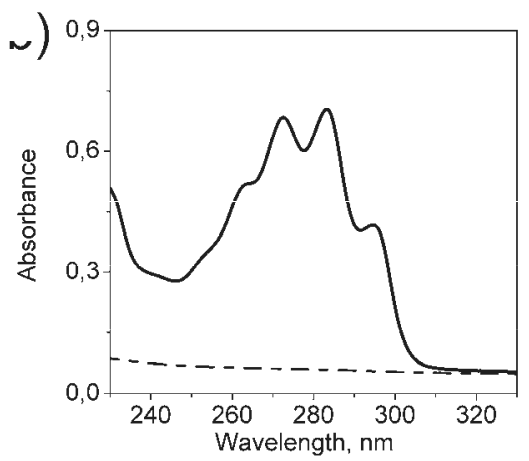

(b)

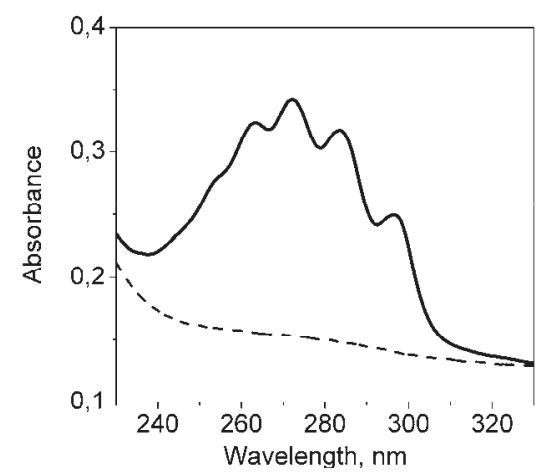

(d)

Figure 1. Absorption spectra of 7-DHC in ethanol (a), in PVB film (b), in silica porous film (c) and in PVA film (d). Dotted lines in Figures 1(b), 1(c) and 1(d) show absorbance of the films without 7-DHC. 


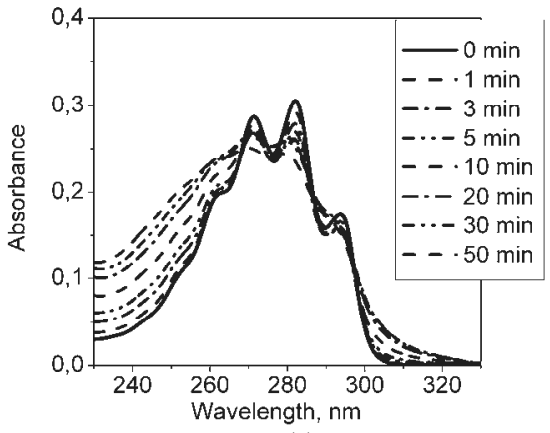

(a)

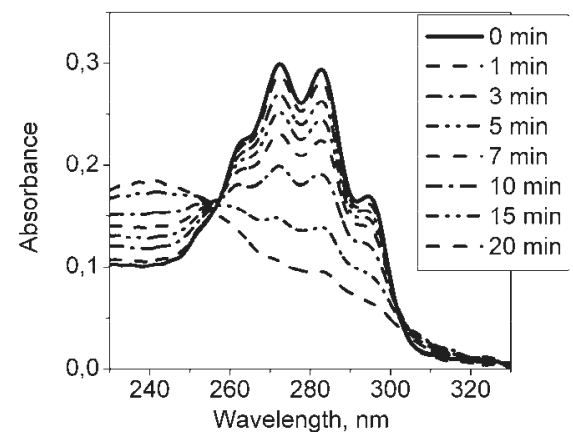

(c)

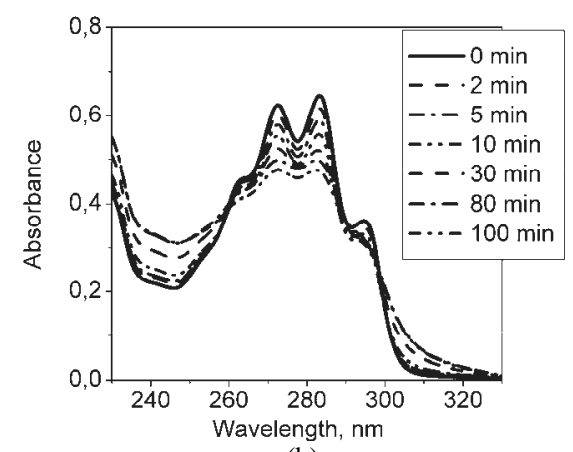

(b)

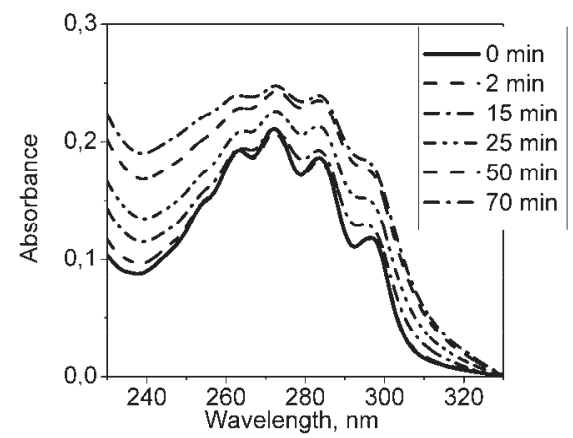

(d)

Figure 3. Transformation of the 7-DHC absorption spectrum in ethanol (a), PVB film (b), silica film (c) and PVA film (d) upon irradiation with the EL-30 UV lamp.
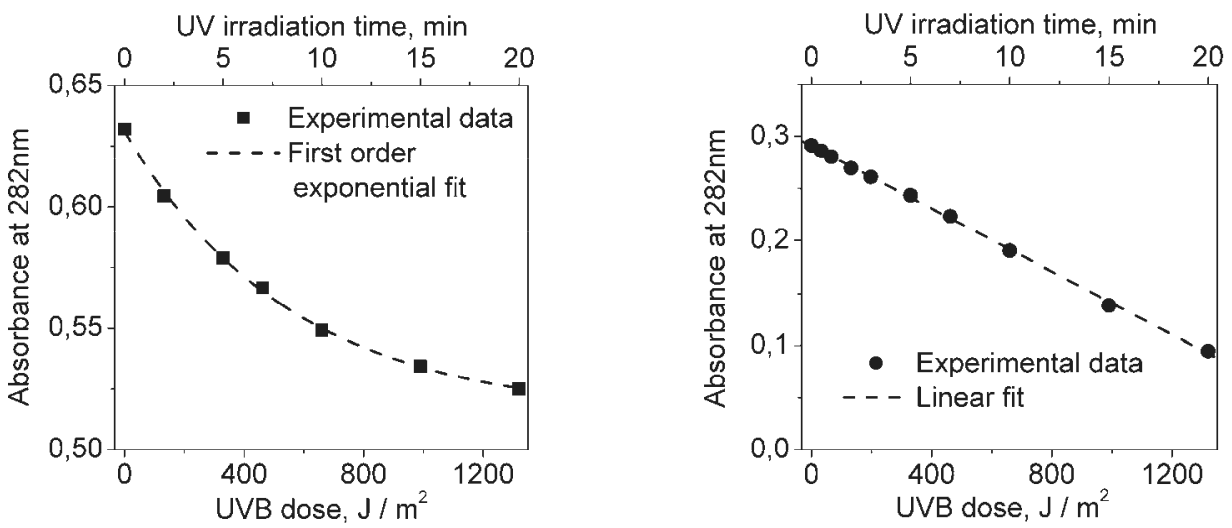

Figure 3. The UVB dose dependence of the 7-DHC absorbance at $282 \mathrm{~nm}$ in PVB polymer film (a) and in silica film (b).

min D), on quartz substrates and free transparent films on the basis of polyvinyl alcohol and polyvinyl butyral doped with 7-DHC. Studies on the effects of UV exposures showed that in view of the complexity of the 7-DHC doped PVA film preparation and, what is more important, nonmonotonic dependence of the absorbance at $282 \mathrm{~nm}$ versus UV exposure, preference should be given to the PVB-based film for personal UV dosimetry.

\section{Acknowledgements}

This research is supported by the GIPP Program of the US Department of Energy (the STCU Project P344).

The authors appreciate helpful advices from Prof.
A. Ischenko (Institute of Organic Chemistry, NAS Ukraine).

\section{REFERENCES}

[1] B. Menaa, M. Takahashi, Y. Tokuda and T. Yoko, "Preparation and Properties of Polyphenylsiloxane-Based Hybrid Glass Films Obtained from a Non-Aqueous Coating Sol Via a Single-Step Dip-Coating," Optical Materials, Vol. 29, No. 7, March 2007, pp. 806-813.

[2] B. Menaa, M. Takahashi, Y. Tokuda and T. Yoko, “A Non Aqueous Route to Optically Active Dyes Rhodamine $6 \mathrm{G}$ and Coumarin 152 Doped in Polyphenylsiloxane,” Solid State Sciences, Vol. 10, No. 9, September 2008, pp. 
1200-1208.

[3] B. Menaa, M. Takahashi, Y. Tokuda and T. Yoko, "Dispersion and Photoluminescence of Free Metal Phtalocyanine in Sol-Gel Glasses," Journal of Photochemistry and Photobiology A, Vol. 194, No. 2-3, February 2008, pp. 362-366.

[4] B. Dunn and J. I. Zink, "Probes of Pore Environment and Molecule-Matrix Interactions in Sol-Gel Materials," Chemistry of Materials, Vol. 9, No. 11, November 1997, pp. 2280-2291.

[5] B. Menaa, F. Menaa, C. Aiolfi-Guimaraes and O. Sharts, "Silica-Based Nanoporous Sol-Gel Glasses: From Bioencapsulation to Protein Folding Studies," International Journal of Nanotechnology, Vol. 7, No. 1, January 2010, pp. 1-45.

[6] B. Menaa, Y. Miyagawa, M. Takahashi, M. Herrero, V. Rives, F. Menaa and D. K. Eggers, "Bioencapsulation of Apomyoglobin in Nanoporous Organosilica Sol-Gel Glasses: Influence of the Siloxane Network on the Conformation and Stability of a Model Protein,” Biopolymers, Vol. 91, No. 11, November 2009, pp. 895-906.

[7] I. P. Terenetskaya, O. G. Perminova and A. M. Eremenko, "Photoisomerization of Provitamin D in Dispersive Systems,” Journal of Molecular Structure, Vol. 219, No. 1-2, March 1990, pp. 359-364.

[8] I. P. Terenetskaya, O. G. Perminova and A. M. Eremenko, "Effect of Environment on the Conformational Equilibrium and Photoconversions of Previtamin D," Journal of Molecular Structure, Vol. 267, No. 4, March 1992, pp.
93-98.

[9] I. P. Terenetskaya, O. G. Dmitrenko and A. M. Eremenko, "Conformational Control in Previtamin D Photochemistry by Heterogeneous Reaction Media," Research on Chemical Intermediates, Vol. 21, No. 6, January 1995, pp. 653-664.

[10] T. N. Orlova and I. P. Terenetskaya, "Specific Features of Photoisomerization of Provitamin $\mathrm{D}_{3}$ in a Nematic Liquid Ctystal,” Optics and Spectroscopy, Vol. 100, No. 4, April 2006, pp. 584-589.

[11] O. N. Galkin and I. P. Terenetskaya, "Vitamin D Biodosimeter: Basic Characteristics and Potential Applications," Journal of Photochemistry and Photobiology B: Biology, Vol. 53, No. 1-3, November 1999, pp.12-19.

[12] I. P. Terenetskaya, "Spectral Monitoring of Biologically Active Solar UVB Radiation Using an In Vitro Model of Vitamin D Synthesis,” Talanta, Vol. 53, No. 1, October 2000, pp. 195-203.

[13] A. G. M. Barret, D. H. R. Barton, R. A. Russell and D. A. Widdowson "Photochemical Transformations. Part 34. Structures of the Toxisterols $s_{2}$," Journal of the Chemical Society Perkin Transactions 1, Vol. 6, March 1977, pp. 631-643.

[14] L. Fizer and M. Fizer, “Steroids,” Mir, Moscow, 1964.

[15] I. B. Berlman, “On an Empirical Correlation between Nuclear Conformation and Certain Fluorescence and Absorption Characteristics of Aromatic Compounds," Journal of Physical Chemistry, Vol. 74, No. 16, August 1970, pp. 3085-3093. 